\title{
Preventing Malaria through Housing Design
}

Malaria is an issue of global importance. This parasitic disease, which is transmitted through the bite of an infected mosquito, currently threatens $44 \%$ of the world's population. In 2013, there were an estimated 198 million infections and over 580,000 deaths from malaria. Like many diseases, malaria is opportunistic, quickly feeding into the cycle of poverty and infecting the most vulnerable members of society who lack access to protection and care. Sadly, it is the young children within these vulnerable populations who shoulder the greatest burden. Among all malaria-related deaths in Africa, which accounts for nine out of ten of all malaria deaths globally, $83 \%$ were among children under the age of 5 years. ${ }^{1}$ The timely control of malaria is a priority, but the tools to achieve this are limited. Growing drug and insecticide resistance threatens our ability to effectively control malaria using currently available methods. As such, the diversification of available strategies is essential for building the required capacity to respond to the complex challenges associated with malaria control in the 21 st century. To achieve this, it is imperative that practitioners from a variety of fields become actively engaged in designing, testing, and implementing high-impact strategies, such as mosquito-proof housing, which can effectively respond to the root causes of the world's most pressing health threats.

\section{CHALLENGES IN MALARIA CONTROL}

Despite over half a million deaths each year, it is important to acknowledge that great strides have been made in the control of this devastating disease. Global mortality has fallen by $47 \%$ since $2000,{ }^{2}$ a success that has been largely due to the rollout of a new drug treatment, known as artemisinin-based combination therapy (ACT), and the global push to expand coverage of insecticide-treated bed nets (ITNs) and indoor residual spraying (IRS). With more lives being saved than ever before, it is easy to view these chemically based strategies as the penultimate step toward the eventual elimination of malaria. However, the persistent over-reliance on a limited array of functionally similar strategies jeopardizes future efforts to control and treat this disease.
OLIVIA YOST

ARCHIVE Global

PETER WILLIAMS

ARCHIVE Global 


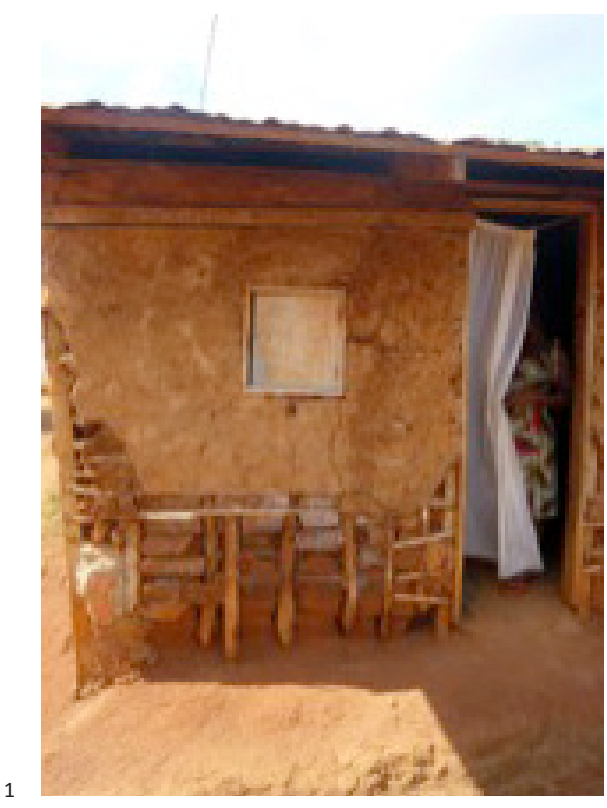

Drug and insecticide resistance is a significant threat. Resistance to artemisinin, the only remaining highly effective drug treatment for malaria, has already been reported in five countries in Southeast Asia. ${ }^{3}$ Additionally, resistance to common insecticide compounds used globally in ITNs and IRS has already been reported in $78 \%$ of 63 surveyed countries. The rapid spread of resistance threatens to reverse past health gains and renders our most powerful tools against malaria obsolete, thereby cutting off our ability to protect and treat the 3.2 billion people currently at risk around the world. ${ }^{4}$

It is also important to note that control strategies like ITN and IRS may not always offer protection as intended. While ITNs are highly successful public health interventions-low-cost, easy to distribute, and efficacious - full protection requires unwavering use by all members of the household. In reality there is often a gap between ITN ownership and actual use, which leaves people exposed to potential mosquito bites and, therefore, infection. The reasons for this disconnect are complex. According to recent research, the most frequently cited reason for lack of regular ITN use was due to discomfort from poor airflow and high temperatures while sleeping under the netting. Furthermore, the failure of the ITN to conform to the family's sleeping patterns and the perception of malaria infection as a low risk were common reasons for net disuse. ${ }^{5,6}$ These findings certainly do not imply that ITNs should be discontinued, but rather highlight clear opportunities to better understand how people choose to interact with their built environment and prioritize health risks. By understanding these variables within the broader context of disease transmission, we can create solutions targeted to extend protection across current gaps.

\section{HOUSING DESIGN AS A STRATEGY FOR MALARIA CONTROL}

The use of housing modifications as a strategy to prevent malaria is not a novel concept. The expansion of higher quality housing in Europe and the United States played a significant role in the reduction of malaria in early 20th century on both continents. ${ }^{7}$ The structure of the home plays a key role in the ecology of malaria transmission, as most Anopheles, the species of mosquito responsible for transmitting malaria, prefer to bite after dusk, when most people are sleeping inside their homes. Due to this specific biting preference, an estimated $80 \%$ of malaria transmission in Africa occurs inside the home. ${ }^{8}$

Inadequately constructed housing allows mosquitoes easy entry into the indoor living environment (Figure 1). Characteristics like open eaves, unscreened windows and doors, mud or thatch walls and roofs, and lack of ceilings are all risk factors for mosquito entry. ${ }^{9,10}$ Simple modifications to home design, such as sealing eaves, directly respond to these unique behavioral characteristics of the mosquito and significantly reduce mosquito entry into the home, thereby decreasing risk of infection for all residents.

Various housing characteristics and design modifications have been tested in the field in order to quantitatively describe the potential for protection against malaria. This protectiveness has been measured in several ways depending on the scope and design of the research. In some cases, mosquito entry into the living environment (i.e. the number of Anopheles mosquitoes inside the home) was used as a measure of risk. For example, a study completed in a highly malarious region of Kenya tested the impact of installing low-cost, locally produced ceilings against mosquito entry into the home. Findings showed a $76-82 \%$ reduction in entry among homes with the modified ceilings, thus demonstrating the potential for the application of simple design modifications to protect residents in high-risk areas ${ }^{11}$ regardless of ITN use. A similar trial, which was completed in the Gambia, also found that the installation of plastic screened ceilings reduced the entry of mosquitoes by $80.1 \%$, when compared to controls. ${ }^{12}$

Figure 1: Example of Cameroonian home with high risk of mosquito entry (ARCHIVE Global, 2013)

Other research has sought to link the quality of home design to actual rates of malaria infection among residents. Research completed in Burkina Faso found that young children living 
in homes with mud roofs had greater than two times the odds of having a malaria infection than children living in homes with iron roofs. ${ }^{13}$ Similar results have been documented in studies from South Asia. Research carried out in Sri Lanka identified significantly higher malaria incidence among residents living in poorly constructed homes, $21.2 \%$, when compared to residents living in adequately constructed homes, $10.5 \%{ }^{14}$

In addition to this research, there has been some limited, yet important, research that assesses residents' receptiveness towards a given mosquito-proofing modification. Upon completion of a trial in the Gambia that tested the efficacy of full home screening, $94 \%$ of participants chose to keep the screening in their homes. Residents cited that the screening not only prevented the entry of mosquitoes, but also improved their privacy and beautified their home. ${ }^{15}$

\section{OPPORTUNITIES FOR IMPACT}

While the initial investment into housing modifications as a malaria prevention strategy may be considerably greater than that of current control strategies like ITNs or IRS, the benefits are significant and potentially more valuable to the user. The function of housing modifications is not dependent on drug resistance and does not require repeated investment over decades or long-term behavior change. Additionally, coverage at the home level is equitable, extending a level of protection to all residents sleeping inside the home regardless of net use (Figure 2). Perhaps most critically, housing modifications may have the power to appeal to the user through the addition of actual and/or perceived value to the home, both financial and sentimental, while simultaneously protecting against a serious health threat that is not necessarily deemed a priority.

By shifting from research to practice, the expansion of high-quality housing can assist in addressing the health challenges that rapidly growing countries in Africa will face in the coming decades. Unprecedented population growth in urban centers will lead to the proliferation of living conditions that will result in a growing burden of diseases associated with poverty. Such a scenario will require cities to care for a greater number of needy residents than every before. Further compounding this issue, the inescapable increase in drug and insecticide resistance will mean that these diseases, like malaria, will be more difficult to treat and control chemically. In the face of these coming challenges, a broader understanding of how housing can be used in conjunction with currently used prevention strategies will be necessary.
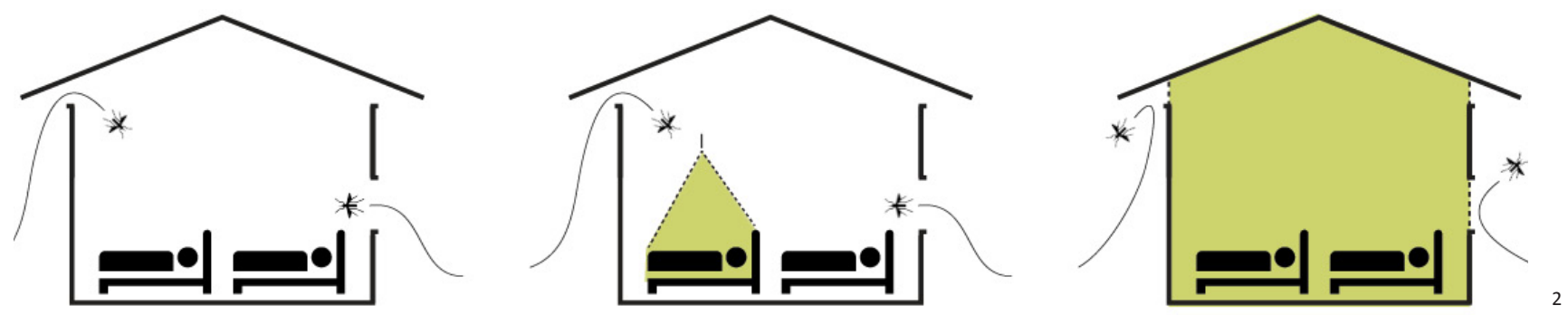

SHIFTING FROM RESEARCH TO PRACTICE: MOSQUITO-PROOF HOUSING IN CAMEROON In response to the clear need for the application of alternative strategies to prevent malaria transmission in vulnerable communities, ARCHIVE Global is working to put these findings into practice in the field. ARCHIVE Global is an international non-profit organization that seeks to reduce the dual burden of poverty and disease through the delivery of housing design support in low-income communities around the world. In addition to the organization's global portfolio of projects, ARCHIVE Global is currently working with the Cameroon

Figure 2: Comparison of protection types. (ARCHIVE Global, 2015) 


\section{ENDNOTES}

1. World Health Organization (WHO). 2014. World Malaria Report 2014. WHO Press: Geneva, Switzerland.

2. WHO, 2014

3. World Health Organization (WHO). 2013. World Malaria Report 2013. WHO Press: Geneva, Switzerland.

4. WHO, 2014

5. Seidlein LV, Ikonomidis K, Bruun R, Jawara M, Pinder M, Knols BGJ, Knudsen JB. 2012. Airflow attenuation and bed net utilization: observations from Africa and Asia. Malaria Journal. 11(200).

6. Pulford J, Hetzel MW, Bryant M, Siba PM, Mueller I. 2011. Reported reasons for not using a mosquito net when one is available: a review of published literature. Malaria Journal. 10(83).

7. Lindsay SW, Emerson PM, Charlwood DJ. 2002. Reducing malaria by mosquito-proofing housings. Trends in Parasitology. 18(11): 510-514.

8. Lindsay, 2002

9. Lwetoijera DW, Kiware SS, Mageni ZD, Dongus S, Harris C, Devine GJ, Majambere S. 2012. A need for better housing to further reduce indoor malaria transmission in areas with high bed net coverage. Parasites and Vectors. 6(57): $1-9$.

10. Yé Y, Hoshen M, Louis V, Séraphin S, Traoré, Sauerborn R. 2006. Housing conditions and Plasmodium falciparum infection: protective effect of iron-sheet roofed houses. Malaria Journal. 5(8): 1-7.
Coalition Against Malaria (CCAM) to design, test, and deliver mosquito-proof housing in the peri-urban community of Minkoameyos, Cameroon. ${ }^{16}$

Malaria remains one of the three main causes of sickness and death in Cameroon. According to findings from the World Health Organization (WHO) in 2010, just $14 \%$ of Cameroonian children under five slept under an ITN every night. ${ }^{17}$ This is paralleled by the high prevalence of poorly constructed housing, particularly in rapidly growing peri-urban settlements. Given this high burden of malaria and poor housing, as well as low ITN use, the application of simple housing modifications as a supplemental prevention measure has the potential to dramatically reduce local transmission, as well as provide practical evidence to inform policy at a national and international level.

ARCHIVE Global and CCAM launched the Building Malaria Prevention (BMP) project in 2013 with the goal of 1) extending mosquito-proof housing to over 260 homes in Minkoameyos, chosen based on a comprehensive selection criteria developed to identify the most at risk homes; and 2) collecting clear evidence of health impact that can be used to demonstrate the value of housing as malaria prevention. The project is comprised of 3 major components: design and construction, community training and advocacy, and malaria research.

As previously mentioned, one of the most common reasons for poor ITN use is discomfort while sleeping. To prevent the similar stagnation of air following the sealing of eaves and other openings, care is taken during the design process to install strategically placed screened windows to passively boost air change within the home and, as a result, maintain a cooler indoor temperature. Other modifications include the sealing of open eaves, installation of screened windows and doors, plastering of cracked walls, and targeted improvement of drainage around the foundation of the home to eliminate potential mosquito breeding sites. Figure 3 depicts a rendering of a completed beneficiary family's home from 2014. Upon completion of the project in December 2015, an estimated 1,500 residents will be living in healthy, mosquito-proof housing and over 4,000 members of the Minkoameyos community will have participated in training workshops that focus on using home design to protect against malaria. ${ }^{18}$

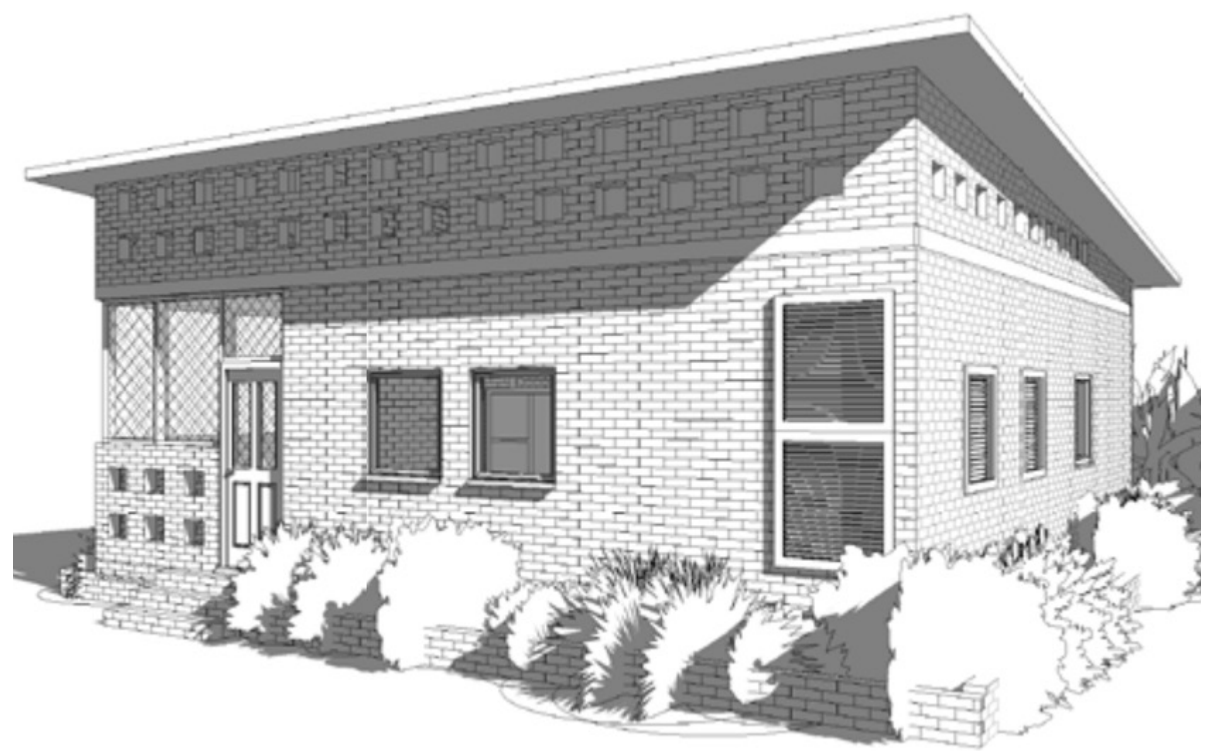

Figure 3: Example of a mosquito-proof housing design (ARCHIVE Global, 2013) 
By rolling out the BMP project in Minkoameyos, ARCHIVE Global and our partners seek to demonstrate the feasibility of implementing this approach at a national and international level though the use of local material, labor, and expertise. The work in Minkoameyos will hopefully lead to broader support for and roll out of similar projects throughout the malaria-affected world. It is clear that repetition and experimentation will be important to refine implementation strategies and to most effectively address the region-specific combinations of housing type, culture, environment, and disease.


\section{CONCLUSION}

As shifts in population growth, living patterns, and climate continue to impact the complex global system in yet unknowable ways, it is increasingly important to apply our limited resources toward designing strategies and policies that respond to root causes of poverty and disease, rather than the symptoms. In the case of global health, attention is often focused on reactionary control measures that so often fail to address the root causes of transmission. Avoiding this outcome will require practitioners to reach across disciplines for solutions that speak to the complexity of the issues being addressed. The fields of architecture, design, and planning will have a fundamental role to play in this process.
11. Atieli H, Menya D, Githeko A, Scott T. 2009. House design modifications reduce indoor resting malaria vector densities in rice irrigation scheme area in western Kenya. Malaria Journal. 8(108): 1-9.

12. Lindsay SW, Jawara M, Paine $K$, Pinder $M$ Walraven GEL, Emerson PM. 2003. Changes in house design reduce exposure to malaria mosquitoes. Tropical Medicine and International Health. 8(6): 512-517.

13. Yé, 2006

14. Gamage-Mendis AC, Carter R, Mendis C, De Zoysa AP, Herath PR, Mendis KN. 1991. Clustering of malaria infections within an endemic population: risk of malaria associated with the type of housing construction. American Journal of Tropical Medicine. 45(1): 77-85

15. Kirby MJ, Ameh D, Bottomley C, Green C, Jawara M, Milligan PJ, Snell PC, Conway DJ, Lindsay SW. 2009. Effect of two different house screening interventions on exposure to malaria vectors and on anaemia in children in The Gambia: a randomised controlled trial. Lancet. 374: 998-1009.

16. ARCHIVE Global. 2015. Building Malaria Prevention. www.archiveglobal.org.

17. World Health Organization. 2010. World Malaria Report 2010. WHO Press: Geneva, Switzerland.

18. ARCHIVE Global, 2015
Figure 4: Beneficiary home pre-renovation

Figure 5: Beneficiary home post-renovation 
